\title{
Potentially Inappropriate Prescriptions of Antipsychotics for Patients With Dementia
}

\author{
Manuel Enrique Machado-Duque 1,2, Luis Fernando Valladales-Restrepo ${ }^{1,2}$, \\ Juan Alberto Ospina-Cano ${ }^{3}$, María José Londoño-Serna ${ }^{3}$ and \\ Jorge Enrique Machado-Alba *1
}

${ }^{1}$ Grupo de Investigación en Farmacoepidemiología y Farmacovigilancia, Universidad Tecnológica de Pereira-Audifarma S.A, Pereira, Colombia, ${ }^{2}$ Grupo de Investigación Biomedicina, Facultad de Medicina, Fundación Universitaria Autónoma de Las Américas, Pereira, Colombia, ${ }^{3}$ Semillero de Investigación en Farmacoepidemiología y Farmacovigilancia, Universidad Tecnológica de Pereira, Pereira, Colombia

\section{OPEN ACCESS}

Edited by: Luciane Cruz Lopes,

University of Sorocaba, Brazil

Reviewed by: Haitham Jahrami,

Arabian Gulf University, Bahrain Victor Florez-Garcia, University of Wisconsin-Milwaukee, United States

*Correspondence: Jorge Enrique Machado-Alba machado@utp.edu.co

Specialty section: This article was submitted to Drugs Outcomes Research and Policies,

a section of the journal Frontiers in Pharmacology

Received: 14 April 2021 Accepted: 17 May 2021 Published: 31 May 2021

Citation: Machado-Duque ME, Valladales-Restrepo $L F$, Ospina-Cano JA, Londoño-Serna MJ and Machado-Alba JE (2021)

Potentially Inappropriate Prescriptions of Antipsychotics for Patients With Dementia. Front. Pharmacol. 12:695315. doi: 10.3389/fphar.2021.695315
Dementias are neurodegenerative and progressive diseases of the central nervous system. The objective of this study was to determine the frequency of potentially inappropriate prescriptions of antipsychotics in a group of patients diagnosed with dementia in Colombia. This was a cross-sectional study based on a population database for drug dispensing that identified prescriptions of antidementia drugs, antipsychotics, and other drugs for patients with a diagnosis of dementia. Descriptive statistics and bivariate and multivariate analyses were performed. A total of 11,372 patients with dementia were identified; $66.6 \%$ were women, and the mean age was $80.5 \pm 9.6$ years. Alzheimer's disease was the most frequent diagnosis (76.6\%). A total of $69.0 \%$ of patients received antidementia drugs. A total of $37.1 \%$ of patients received some antipsychotic, especially atypical antipsychotics (31.0\%). Increased age, being treated with memantine, simultaneously presenting with anxiety, depression, and psychotic disorders, and concomitantly receiving anticonvulsants, bronchodilators and benzodiazepines were associated with a greater probability of being prescribed antipsychotics. More than one-third of patients with dementia received antipsychotic prescriptions, which are considered potentially inappropriate because they can worsen cognitive decline and favor the occurrence of adverse events.

Keywords: (MeSH): dementia, alzheimer disease, dementia vascular, antipsychotic agents, pharmacoepidemiology

\section{INTRODUCTION}

Dementia is a neurodegenerative and progressive disease of the central nervous system characterized by chronic, global and generally irreversible deterioration of cognitive ability (Prince et al., 2013). Dementia may be due to a variety of underlying pathophysiological processes; the most common is Alzheimer's disease (50-75\%), followed by vascular dementia (20\%), dementia with Lewy bodies (5\%), and frontotemporal dementia (5\%) (Cunningham et al., 2015).

Global rates of dementia are increasing rapidly, in line with population aging. The prevalence of dementia is $2-3 \%$ in the $70-75$ age group, increasing to $25 \%$ in those over 85 (Rizzi et al., 2014). At advanced ages, women are more likely to develop dementia due to Alzheimer's disease, while the prevalence of vascular dementia is higher in men (Rizzi et al., 2014). 
The pathophysiological processes underlying dementia are not yet fully understood; however, it is known that all involve pathological protein accumulation (Kocahan and Doğan, 2017). These pathological accumulations are associated with synaptic failure, neuronal loss and atrophy that can have different anatomical distribution patterns (Frisoni et al., 2015).

Neuropsychiatric symptoms, including agitation, aggressiveness, paranoid delusions, and hallucinations, are widely prevalent among dementias, especially in the moderate and severe disease stages, which require either pharmacological or nonpharmacological intervention (Cerejeira et al., 2012). Very often, nonpharmacological management is sufficient to control symptoms, but sometimes, the severity of the disorder, the involvement in the patient, or the risk to oneself or others makes using drugs, including antipsychotics, necessary to control symptoms (Calsolaro et al., 2021). However, the Beers criteria and the STOPP/START criteria consider antipsychotics as potentially inappropriate prescriptions in this group of patients because they are associated with a greater number of adverse outcomes, such as cerebrovascular events and greater functional decline and mortality, and thus recommend avoiding their use unless there are no alternatives for the management of behavioral disorders or delirium ("American Geriatrics Society 2019 Updated AGS Beers Criteria(R) for Potentially Inappropriate Medication Use in Older Adults," 2019; O’Mahony et al., 2015).

It has been shown that patients treated with antipsychotics can frequently present with anticholinergic side effects, such as orthostatic hypotension, confusion, drowsiness, cognitive impairment, and an increased risk of severe extrapyramidal effects that can be fatal (Schneider et al., 2006a; Schneider et al., 2006b; Calsolaro et al., 2021; Vina Latin Small Letter et al., 2021). In addition, the DART-AD trial found an increased risk of mortality in patients older than 65 years with dementia treated with any class of antipsychotics, both in the short term (12 weeks) and long term (over 48 months) (Hereu and Vallano, 2011).

The inadequate use of antipsychotics and the lack of precautions in their prescription for Colombian patients with different types of dementia are unknown. Therefore, the objective of this study was to determine the frequency of potentially inappropriate prescriptions of antipsychotics in a group of patients diagnosed with dementia in Colombia between October and December of 2019, as well as to identify the medications used, comorbidities and possible variables associated with the use of these antipsychotics in this older adult population.

\section{MATERIALS AND METHODS}

\section{Study Design and Patients}

A cross-sectional study was conducted on the prescription patterns of antipsychotic drugs for patients diagnosed with dementia in out-patient setting. The data were obtained from a population database for drug dispensing that contains information on approximately 8.5 million people affiliated with the Health System of Colombia through six health insurance companies, corresponding to approximately $30.0 \%$ of the active affiliated population covered by the contributory or paid regime and $6.0 \%$ of the population covered by the statesubsidized regime, which serves $17.3 \%$ of the Colombian population.

Patients aged 50 years or older of either sex and seen as outpatients were selected in the main cities of Colombia, with a diagnosis of dementia who received a drug prescription in any month in the period from October 1 to December 31, 2019, were included (Medication for the treatment of any comorbidity or dementia in which an International Classification of Diseases (ICD-10) diagnostic code for dementia is used or registered). Identification was performed based on ICD-10 codes, considering the following diagnoses: Alzheimer's disease (F000-F002, F009, G300, G301, G308 and G309), vascular dementia (F010-F013, F018 and F019), dementia in Parkinson's disease (F023), dementia in Huntington's disease (F022), dementia in Pick's disease or frontotemporal dementia (F020), dementia in HIV (F024), dementia in Creutzfeldt-Jakob disease (F021) and unspecified dementias (F03X and F028).

The information of each dispensing and variables associated with the prescription is obtained from the pharmacies and the clinical registry, they are kept in a database with all the information and subsequently consulted through business intelligence, validated by validated by two members of the research group, identifying strange and missing values, until having a dataset with the included patients and being able to analyze it, and subsequently analyzed. Their drug dispensing records during the observation period were analyzed to identify the use of antipsychotics and all comedications. Those with incomplete information and those with two or more different dementia diagnoses were excluded.

\section{Variables}

Based on the information on the consumption of drugs of the affiliated population, systematically obtained by the dispensing company (Audifarma SA), a database was designed that allowed collecting the following groups of variables: 1). Sociodemographic: sex, age, city, and geographic region of residence; 2). Clinical: type of dementia diagnosed and comorbidities. 3). Comorbidities: identified from the diagnoses reported using ICD-10 codes for the selected patients. They were categorized by number of comorbidities. The main cardiovascular, endocrine, rheumatologic, urological, renal, psychiatric, neurological, digestive, respiratory, and cancer diseases were identified; 4). Drugs for dementia treatment: acetylcholinesterase inhibitors (rivastigmine, donepezil, galantamine) and NMDA receptor antagonists (memantine); 5). Prescribed antipsychotics: Typical: chlorpromazine, fluphenazine, haloperidol, levomepromazine, pipotiazine, propericiazine, thioridazine, and trifluoperazine; and Atypical: amisulpride, aripiprazole, asenapine, clozapine, olanzapine, paliperidone, quetiapine, risperidone, sulpiride, and ziprasidone; and 6). Comedications were grouped into the following categories: 1) antidiabetics (oral and subcutaneous), 2) antihypertensives and diuretics, 3) lipid-lowering drugs; 4) antiulcers, 5) antidepressants, 6) benzodiazepines, 7) thyroid 
hormone, 8) anticonvulsants, 9) antiarrhythmics, 10) antihistamines, 11) bronchodilators and inhaled corticosteroids, 12) opioid analgesics, 13) nonopioid analgesics, 14) platelet antiaggregants, and 15) anticoagulants.

\section{Ethical Statement}

The protocol was approved by the Bioethics Committee of Universidad Tecnológica de Pereira in the "research without risk" category (Code: 02-130420). The ethical principles established by the Declaration of Helsinki were respected. No personal data were collected from the patients.

\section{Data Analysis}

The data were analyzed with the statistical package SPSS Statistics, version 26.0 for Windows (IBM, United States). A descriptive analysis was performed; qualitative variables are presented as frequencies and proportions, and quantitative variables are presented as measures of central tendency and dispersion. Quantitative variables were compared using Student's t-test or ANOVA, and categorical variables were compared using the $\chi^{2}$ test. Binary logistic regression models were fitted using "prescribed antipsychotics" as the dependent variable; the covariates were the variables that were significantly associated with the dependent variable in the bivariate analyses and variables with biological plausibility to explain the outcome (prescription of an antipsychotic). A statistical significance level of $p<0.05$ was adopted.

\section{RESULTS}

A total of 11,372 patients with a diagnosis of dementia were identified, distributed in 154 different cities or municipalities. The mean age was $80.5 \pm 9.6$ years (range: $50.0-105.1$ years), and $66.6 \%(n=7,573)$ were women. A total of $78.8 \%(n=8,958)$ of the patients resided in capital cities, and the majority were in the Pacific Region ( $n=3,009 ; 26.5 \%$ ), followed by the BogotáCundinamarca Region ( $n=2,982 ; 26.2 \%)$, Caribbean Region ( $n=2,758 ; 24.3 \%)$, Central Region $(n=2081 ; 18.3 \%)$, Eastern Region $(n=519 ; 4.6 \%)$ and Amazonia-Orinoquía Region $(n=$ 23; $0.2 \%)$.

Most patients had a diagnosis of Alzheimer's disease $(n=$ $8,711 ; 76.6 \%)$, followed by unspecified dementia ( $n=1767$; $15.5 \%)$, vascular dementia $(n=657 ; 5.8 \%)$, dementia in Parkinson's disease $(n=201 ; 1.8 \%)$, frontotemporal dementia $(n=21 ; 0.2 \%)$, dementia in Huntington's disease $(n=9 ; 0.1 \%)$ and dementia in HIV $(n=6 ; 0.1 \%)$. A total of $69.0 \%(n=7,841)$ were receiving pharmacological treatment for dementia, mainly rivastigmine $(n=4,843 ; 42.6 \%)$, followed by memantine $(\mathrm{n}=$ $3,756 ; 33.0 \%)$, donepezil $(n=164 ; 1.4 \%)$ and galantamine $(n=$ $164 ; 1.4 \%)$.

Of the total number of patients identified, 4,222 (37.1\%) had been prescribed at least one antipsychotic, distributed among 10 different antipsychotic drugs, with the use of atypical antipsychotics $(n=3,526,31.0 \%)$ predominating over the use of typical antipsychotics $(n=1,023,9.0 \%)$, with some patients receiving more than one of these drugs. Of the patients who were
TABLE 1 | Antipsychotics prescriptions for a group of patients with dementia in Colombia, 2019.

\begin{tabular}{lcc}
\hline Antipsychotic & Frequency $\boldsymbol{n}=\mathbf{1 1 , 3 7 2}$ & \% \\
\hline Atypical & 3,526 & 31.0 \\
Quetiapine (tablet) & 2,686 & 23.6 \\
Risperidone & 525 & 4.6 \\
Tablet & 399 & 3.5 \\
Oral solution & 120 & 1.1 \\
Injectable & 12 & 0.1 \\
Clozapine (tablet) & 312 & 2.7 \\
Olanzapina (tablet) & 185 & 1.6 \\
Aripiprazol (tablet) & 17 & 0.1 \\
Paliperidone & 10 & 0.1 \\
Injectable & 9 & 0.1 \\
Tablet & 1 & 0.0 \\
Amisulpiride (tablet) & 1 & 0.0 \\
Typical & 1,023 & 9.0 \\
Levomepromazine & 600 & 5.3 \\
Oral solution & 541 & 4.8 \\
Tablet & 63 & 0.6 \\
Haloperidol & 489 & 4.3 \\
Oral solution & 438 & 3.9 \\
Injectable & 57 & 0.5 \\
Tablet & 27 & 0.2 \\
Pipothiazine (injectable) & 6 & 0.1 \\
& &
\end{tabular}

prescribed antipsychotics, $86.6 \%(n=3,656)$ received a single drug, and $13.4 \%(n=566)$ received two or more. The most commonly used antipsychotic was quetiapine, followed by levomepromazine and risperidone. The most commonly used pharmaceutical forms were tablets $(n=3,482 ; 30.6 \%)$, followed by oral solution $(n=1,026 ; 9.0 \%)$ and injectable solution $(n=82$; $0.7 \%$ ) (Table 1).

\section{Comorbidities and Comedications}

A total of $77.8 \%(n=8,853)$ of all patients had some chronic disease. Of these, $42.9 \%(n=3,805)$ had one disease, $31.8 \%(n=$ $2,812)$ had two diseases and $25.3 \%(n=2,236)$ had three or more diseases. The 10 comorbidities that were most common in all patients were arterial hypertension $(n=4,922 ; 43.3 \%)$, diabetes mellitus ( $n=1,547 ; 13.6 \%)$, urinary incontinence $(n=1,530$; $13.5 \%)$, bipolar affective disorder $(n=993 ; 8.7 \%)$, anxiety disorders $(n=751 ; 6.6 \%)$, hypothyroidism ( $n=769 ; 6.8 \%)$, depressive disorders $(n=751 ; 6,6 \%)$, chronic kidney disease $(n=643 ; 5.7 \%)$, chronic obstructive pulmonary disease $(n=465$; $4.1 \%)$ and benign prostatic hyperplasia $(n=434 ; 3.8 \%)$. Upon grouping, cardiovascular diseases were the most frequent $(n=$ $5,169 ; 45.5 \%)$, followed by other psychiatric disorders $(n=2,644$; $23.3 \%)$ and endocrine $(n=2,470 ; 21.7 \%)$, urological $(n=2029$; $17.8 \%)$, neurological $(n=1,059 ; 9.3 \%)$, rheumatic $(n=776,6.8 \%)$, gastrointestinal $(n=602 ; 5.3 \%)$ and respiratory $(n=501 ; 4.4 \%)$ diseases.

The most prescribed comedications were antihypertensives and diuretics $(n=6,902,60.7 \%)$, followed by antidepressants $(n=$ $5,198 ; 45.7 \%)$, nonopioid analgesics $(n=3,696 ; 32.5 \%)$, antiulcers $(n=3,384 ; 29.8 \%)$, platelet antiaggregants $(n=3,125 ; 27.5 \%)$, lipid-lowering drugs $(\mathrm{n}=2,915 ; 25.6 \%)$, thyroid hormone $(n=$ $2,558 ; 22.5 \%)$, oral and subcutaneous antidiabetic drugs and 
TABLE 2 | Comparison of sociodemographic, clinical and pharmacological variables with types of dementia in a group of patients in Colombia, 2019.

\begin{tabular}{|c|c|c|c|c|c|c|c|c|c|c|}
\hline \multirow[t]{2}{*}{ Variables } & \multicolumn{2}{|c|}{ Alzheimer's disease } & \multicolumn{2}{|c|}{$\begin{array}{l}\text { Unspecified } \\
\text { dementias }\end{array}$} & \multicolumn{2}{|c|}{$\begin{array}{l}\text { Vascular } \\
\text { dementia }\end{array}$} & \multicolumn{2}{|c|}{$\begin{array}{c}\text { Dementia in } \\
\text { Parkinson's } \\
\text { disease }\end{array}$} & \multicolumn{2}{|c|}{ Other dementias } \\
\hline & $n=8,711$ & $\%$ & $n=1767$ & $\%$ & $n=657$ & $\%$ & $n=201$ & $\%$ & $n=36$ & $\%$ \\
\hline Woman & 5,945 & 68.2 & 1,151 & 65.1 & 375 & 57.1 & 87 & 43.3 & 15 & 41.7 \\
\hline Age (mean; SD) & \multicolumn{2}{|c|}{$80.7 \pm 9.5$} & \multicolumn{2}{|c|}{$80.5 \pm 10.1$} & \multicolumn{2}{|c|}{$80.9 \pm 9.7$} & \multicolumn{2}{|c|}{$77.7 \pm 9.5$} & \multicolumn{2}{|c|}{$68.4 \pm 9.6$} \\
\hline Geographic regions & - & - & - & - & - & - & - & - & - & - \\
\hline Pacific region & 2045 & 23.2 & 599 & 33.9 & 299 & 45.5 & 75 & 37.3 & 11 & 30.6 \\
\hline Bogotá-cundinamarca region & 2,304 & 26.4 & 523 & 29.6 & 110 & 16.7 & 34 & 16.9 & 11 & 30.6 \\
\hline The caribbean region & 2,332 & 26.8 & 297 & 16.8 & 83 & 12.6 & 36 & 17.9 & 10 & 27.8 \\
\hline Central region & 1,591 & 18.3 & 298 & 16.9 & 145 & 22.1 & 46 & 22.9 & 1 & 2.8 \\
\hline Eastern region & 444 & 5.1 & 46 & 2.6 & 17 & 2.6 & 9 & 4.5 & 3 & 8.3 \\
\hline Amazon region-orinoquía & 15 & 0.2 & 4 & 0.2 & 3 & 0.5 & 1 & 0.5 & 0 & 0.0 \\
\hline With chronic comorbidities & 6,748 & 77.5 & 1,349 & 76.3 & 559 & 85.1 & 170 & 84.6 & 27 & 75.0 \\
\hline 1 pathology & 2,914 & 33.5 & 595 & 33.7 & 232 & 35.3 & 52 & 24.9 & 12 & 33.3 \\
\hline 2 pathologies & 2,132 & 24.5 & 426 & 24.1 & 175 & 23.1 & 70 & 34.8 & 9 & 25.0 \\
\hline$\geq 3$ pathologies & 1702 & 19.5 & 328 & 18.6 & 152 & 23.1 & 48 & 23.9 & 6 & 16.7 \\
\hline Cardiovascular pathologies & 3,998 & 45.9 & 776 & 43.9 & 314 & 47.8 & 71 & 35.3 & 10 & 27.8 \\
\hline Psychiatric pathologies & 2013 & 23.1 & 407 & 23.0 & 155 & 23.6 & 56 & 27.9 & 13 & 36.1 \\
\hline Endocrine pathologies & 1924 & 22.1 & 365 & 20.7 & 147 & 22.4 & 30 & 14.9 & 4 & 11.1 \\
\hline Urological pathologies & 1,533 & 17.6 & 285 & 16.1 & 159 & 24.2 & 47 & 23.4 & 5 & 13.9 \\
\hline Neurological pathologies & 696 & 8.0 & 170 & 9.6 & 95 & 14.5 & 91 & 45.3 & 7 & 19.4 \\
\hline Use of antidementiants & 6,571 & 75.4 & 897 & 50.8 & 314 & 47.8 & 50 & 24.9 & 9 & 25.0 \\
\hline Rivastigmine & 3,917 & 45.0 & 686 & 38.8 & 192 & 29.2 & 42 & 20.9 & 6 & 16.7 \\
\hline Memantine & 3,372 & 38.7 & 238 & 13.5 & 134 & 20.4 & 8 & 4.0 & 4 & 11.1 \\
\hline Donepezil & 146 & 1.7 & 13 & 0.7 & 5 & 0.8 & 0 & 0.0 & 0 & 0.0 \\
\hline Galantamine & 134 & 1.5 & 21 & 1.2 & 8 & 1.2 & 1 & 0.5 & 0 & 0.0 \\
\hline Use of antipsychotics & 3,139 & 36.0 & 712 & 40.3 & 279 & 42.5 & 70 & 34.8 & 22 & 61.1 \\
\hline Atypical & 2,680 & 30.8 & 537 & 30.4 & 226 & 34.4 & 66 & 32.8 & 17 & 47.2 \\
\hline Quetiapine & 2031 & 23.3 & 416 & 23.5 & 178 & 27.1 & 49 & 24.4 & 12 & 33.3 \\
\hline Risperidone & 447 & 5.1 & 52 & 2.9 & 18.0 & 2.7 & 6 & 3.0 & 2 & 5.6 \\
\hline Clozapine & 217 & 2.5 & 61 & 3.5 & 21 & 3.2 & 10 & 5.0 & 3 & 8.3 \\
\hline Typical & 705 & 8.1 & 232 & 13.1 & 74 & 11.3 & 5 & 2.5 & 7 & 19.4 \\
\hline Levomepromazine & 427 & 4.9 & 127 & 7.2 & 37 & 5.6 & 5 & 2.5 & 4 & 11.1 \\
\hline Haloperidol & 314 & 3.6 & 129 & 7.3 & 42 & 6.4 & 1 & 0.5 & 3 & 8.3 \\
\hline Pipothiazine & 4 & 0.0 & 2 & 0.1 & 0 & 0.0 & 0 & 0.0 & 0 & 0.0 \\
\hline Comedications & - & - & - & - & - & - & - & - & - & - \\
\hline Antihypertensives and diuretics & 5,267 & 60.5 & 1,078 & 61.0 & 439 & 66.8 & 103 & 51.2 & 15 & 41.7 \\
\hline Antidepressants & 3,922 & 45.0 & 851 & 48.2 & 321 & 48.9 & 88 & 43.8 & 16 & 44.4 \\
\hline Non-opioid pain relievers & 2,786 & 32.0 & 593 & 33.6 & 228 & 34.7 & 77 & 38.3 & 12 & 33.3 \\
\hline Antiulcer & 2,517 & 28.9 & 572 & 32.4 & 214 & 32.6 & 67 & 33.3 & 14 & 38.9 \\
\hline Platelet antiaggregants & 2,353 & 27.0 & 472 & 26.7 & 250 & 38.1 & 44 & 21.9 & 6 & 16.7 \\
\hline
\end{tabular}

*Other dementias: Includes dementia in Huntington's disease, frontotemporal dementia, and dementia in human immunodeficiency virus infection.

insulins ( $n=2004 ; 17.6 \%)$, anticonvulsants $(n=1,491 ; 13.1 \%)$ and bronchodilators and inhaled corticosteroids $(n=$ $1,416 ; 12.5 \%)$.

\section{Bivariate Comparisons by Type of Dementia}

Alzheimer's disease, unspecified dementia and vascular dementia predominated in women and occurred at a higher mean age. Patients with vascular dementia and Parkinson's disease presented with comorbidities with greater frequency. Rivastigmine and memantine were the most commonly used antidementia drugs for all types of dementia, especially Alzheimer's disease. The prescription of antipsychotics was more common for patients with vascular dementia, with the use of atypical antipsychotics prevailing for all types of dementia; the lowest frequencies of use of typical antipsychotics were found for patients with dementia in Parkinson's disease. The use of antihypertensives and diuretics predominated for all patients, while platelet antiaggregants were prescribed more for patients with vascular dementia (Table 2).

\section{Multivariate Analysis}

Binary logistic regression analysis was performed to determine the factors associated with the use of antipsychotics for patients diagnosed with dementia. Female sex, diagnosis of Alzheimertype dementia, and being treated with rivastigmine decreased the probability of being prescribed an antipsychotic. Increased age, being treated with memantine, presenting concomitantly with anxiety, depression, and psychotic disorders, and receiving 
TABLE 3 | Multivariate analysis of the variables associated with the prescription of antipsychotics for a group of patients with dementia in Colombia, 2019.

\begin{tabular}{|c|c|c|c|c|}
\hline \multirow[t]{2}{*}{ Variable } & \multirow[t]{2}{*}{$p$} & \multirow[t]{2}{*}{ ORa } & \multicolumn{2}{|c|}{$95 \% \mathrm{Cl}$} \\
\hline & & & Lower & Upper \\
\hline Female gender & $<0.01$ & 0.814 & 0.745 & 0.889 \\
\hline Age less than 65 years & Ref & Ref & & \\
\hline Age between 65 and 74 years & $<0.01$ & 1.398 & 1.153 & 1.694 \\
\hline Age between 75 and 84 years & $<0.01$ & 1.656 & 1.388 & 1.975 \\
\hline Age greater than or equal to 85 years & $<0.01$ & 1.916 & 1.604 & 2.29 \\
\hline Be treated in Bogotá D.C/Cundinamarca & 0.004 & 0.856 & 0.771 & 0.95 \\
\hline Diagnosis of Alzheimer's dementia & 0.009 & 0.86 & 0.769 & 0.963 \\
\hline Being treated for dementia with rivastigmine & $<0.01$ & 0.835 & 0.764 & 0.913 \\
\hline Being treated for dementia with memantine & 0.017 & 1.121 & 1.021 & 1.232 \\
\hline Anxiety (comorbidity) & $<0.01$ & 13.107 & 10.783 & 15.93 \\
\hline Depression (comorbidity) & $<0.01$ & 2.184 & 1.853 & 2.574 \\
\hline Osteoporosis (comorbidity) & 0.014 & 0.661 & 0.475 & 0.919 \\
\hline Psychotic disorders (comorbidity) & $<0.01$ & 10.273 & 6.961 & 15.162 \\
\hline Concomitant use of antiepileptic drugs & $<0.01$ & 1.9 & 1.686 & 2.142 \\
\hline Concomitant use of benzodiazepines & $<0.01$ & 1.946 & 1.644 & 2.302 \\
\hline Concomitant use of bronchodilators & 0.001 & 1.237 & 1.092 & 1.403 \\
\hline Concomitant use of lipid-lowering drugs (statins) & $<0.01$ & 0.839 & 0.761 & 0.924 \\
\hline
\end{tabular}

Binary logistic regression: adjusted for sex, age, place of treatment, type of dementia, treatment of dementia, comorbidities, and comedications. ORa, Adjusted Odds Ratio; Cl95\%, Confidence interval.

anticonvulsants, bronchodilators and benzodiazepines simultaneously were associated with a greater probability of being prescribed antipsychotics (Table 3 ).

\section{DISCUSSION}

The present study was able to determine the prescription and the potentially inappropriate use of antipsychotics for patients diagnosed with dementia in six health insurance entities in Colombia. The results of this study are of great interest because a high frequency of use of antipsychotics was found in this specific group of patients, who may be at risk of adverse events. With these results, a baseline for the problem is established, which may be useful for making better informed decisions and improving the use of these drugs.

The mean age of the analyzed patients was close to 80 years, consistent with what has been described in different studies conducted worldwide, i.e., a typical age of presentation of dementias above 65 years (Prince et al., 2013; Arvanitakis et al., 2019). Regarding the sex of the patients, approximately two-thirds of the study population were women, which is also consistent with what has been described in the world population (Goodman et al., 2017; Valladales-Restrepo et al., 2019). The most prevalent type of dementia was Alzheimer's disease, a finding that agrees with reports on the prevalence of dementia worldwide and imparts confidence and validity to the results of this study (Cunningham et al., 2015). Additionally, the most commonly used antidementia drug in the evaluated patients was rivastigmine, agreeing with the data reported in different studies (Jia et al., 2016; Calvo-Torres et al., 2019). In addition, a diagnosis of Alzheimer-type dementia acted as a protective factor for the prescription of antipsychotics, unlike that seen for other types of dementia, a finding that may be explained by the difference in the frequency of psychotic symptoms, agitation and insomnia among the different causes of dementia (Collins et al., 2020).

It was found that more than one-third of patients diagnosed with dementia were prescribed at least one antipsychotic, putting these patients at particular risk of adverse reactions and worsening of symptoms, a result that is similar to those reported in studies conducted in Norway by Langballe et al. (2013) and in Germany by Schulze et al. (2012) (Schulze et al., 2013; Langballe et al., 2014), who found a frequencies of use of 30.4 and $39.8 \%$, respectively. Additionally, it is noteworthy that there was a predominance of prescriptions for atypical antipsychotics over typical antipsychotics, a relationship that contrasts with results from studies by Schulze et al. and Langballe et al., in which 24.4 and $19.5 \%$ of patients with dementia received typical antipsychotics, respectively (Schulze et al., 2013; Langballe et al., 2014).

The most commonly used antipsychotic was quetiapine; in contrast, in the United States, the use of olanzapine predominates, and in Norway, the use of risperidone predominates (Kamble et al., 2009; Langballe et al., 2014; Maust et al., 2015). This trend in Colombia may be due to the off-label use of quetiapine as a sedative-hypnotic (Dolder and McKinsey, 2010).

Cardiovascular disease was the most prevalent comorbidity in almost half of patients with Alzheimer's disease. It has been described that the presence of systolic hypertension can be a modifiable risk factor for the onset of cognitive decline and vascular dementia and that its control with antihypertensive treatment reduces the development of Alzheimer's disease, as well as of other dementias, in the future (Gorelick et al., 2011). In addition, it is noteworthy that coronary and atherosclerotic carotid disease are risk factors for the onset of cognitive decline and vascular dementia (Rosano et al., 2005; Zhong et al., 2012). 
The association of having a diagnosis of osteoporosis with a lower probability of being prescribed an antipsychotic in this group of patients with dementia contrasts with the results of a study by Crews et al. (2012), which found a significant relationship between the use of antipsychotics and a decrease in bone mineral density (Crews and Howes, 2012) as well as a slightly higher risk of having a hip fracture (Jalbert et al., 2010), which should serve as an alert to avoid their use in these patients. In turn, the use of lipid-lowering drugs was also associated with a lower risk of receiving antipsychotics, which supports the findings by Shen et al., who showed that statins can help in the treatment of schizophrenia, based on reduced scores on the positive symptoms, negative symptoms and general subscales of the Positive and Negative Syndrome Scale (PANNS) (Shen et al., 2018). However, the mechanism by which statins reduce psychotic symptoms in these patients is still unclear, but it has been proposed that they can also protect patients with dementia from the onset of this type of manifestation (Shen et al., 2018).

There are reports of a possible association between pulmonary diseases and an increased risk of developing dementia (Liao et al., 2015; Peng et al., 2015). The use of short-acting bronchodilators, such as salbutamol and terbutaline, can cause symptoms such as anxiety, insomnia, motor restlessness and agitation, which may provide an explanation for the greater probability of patients who also have dementia being prescribed antipsychotics (Román, 2011).

The difference found for patients who received rivastigmine, who showed a lower probability of being prescribed an antipsychotic, may be because rivastigmine is used for patients with mild to moderate dementia (Cruz Jentoft and Hernández, 2014), whereas the use of memantine, whose indication of use is for more severe forms of the disease, may imply that patients are more symptomatic and therefore require antipsychotics (Fink et al., 2020).

In this study, it was found that having a concomitant diagnosis of dementia and anxiety markedly increases the likelihood of being prescribed an antipsychotic despite the risks associated with them, making the surveillance, symptom control and appropriate use of psychotropic drugs more important. In addition to the high frequency of comorbidities, studies conducted in people with dementia living in the community found that many behavioral symptoms are closely related to anxiety (Ferretti et al., 2001; Neville and Teri, 2011).

An expected finding was the relationship between psychotic disorders and being prescribed an antipsychotic, although all these drugs are considered potentially inappropriate prescriptions in patients with dementia, with the exception of cases in which there are no alternatives due to severe behavioral and psychotic symptoms (Magierski et al., 2020). The association between dementia and depression with a greater probability of being prescribed an antipsychotic has also been reported (Neil et al., 2003; Nemeroff, 2005; Byers and Yaffe, 2011). In this analysis, more than $45 \%$ of patients with dementia received some antidepressant, a finding that should incite clinicians to consider the need to add, or not, new drugs to this group of patients and the risks involved.
In this study, it was found that the concomitant use of anticonvulsants increased, by almost two-fold, the probability of being prescribed an antipsychotic, a result that may be due to the need to share the same objective to modulate symptoms such as anxiety, depression, apathy, and psychosis and control the behavioral symptoms of patients with dementia who do not respond to typical therapy; however, it cannot be ruled out that anticonvulsants are indicated for the control of seizures (Magierski et al., 2020).

In addition to anticonvulsants, the concomitant use of benzodiazepines seems to double the probability of being prescribed an antipsychotic; however, in this study, due to its cross-sectional observational nature, it cannot be established which was used first. Benzodiazepines are useful in the control of symptoms such as aggressiveness, sleep disturbances and anxiety, which may be associated with the fact that these patients have more behavioral disturbances (Donoghue and Lader, 2010; Dell'osso and Lader, 2013), but it cannot be ruled out that these drugs were used for indications such as panic attacks or anxiety disorders. However, the chronic use of benzodiazepines can worsen behavioral problems due to their amnesic and disinhibitory effects (Rayner et al., 2006).

This analysis has limitations characteristic of observational studies performed using a drug dispensing database. There was a lack of a review of medical records to verify the severity and time since onset of dementia and hence the need for certain drugs. The effectiveness of the therapy and the safety problems that were actually generated with the prescription of antipsychotics were also not evaluated. However, the results can be extrapolated to populations with similar insurance status and drug access characteristics.

Based on the above findings, it can be concluded that more than one-third of this group of Colombian patients diagnosed with dementia were being prescribed some antipsychotic that could potentially worsen their clinical picture and cause adverse events. The progressive increase in age, concomitant suffering from anxiety, depression or some psychotic disorder, and combined use with anticonvulsants and bronchodilators were associated with a greater probability of being prescribed an antipsychotic. Our findings can be used by clinicians and all those responsible for the care of patients with dementia to offer therapies with lower risks to these specific groups. Further studies are needed to explore the safety of these drugs in the context of real life.

\section{DATA AVAILABILITY STATEMENT}

The database with all the information necessary to carry out the analyzes is deposited in protocols.io, https://doi.org/10.17504/ protocols.io.bt7qnrmw.

\section{ETHICS STATEMENT}

The protocol was approved by the Bioethics Committee of Universidad Tecnológica de Pereira in the "research without risk" category. The ethical principles established by the Declaration of 
Helsinki were respected. No personal data were collected from the patients. Written informed consent for participation was not required for this study in accordance with the national legislation and the institutional requirements.

\section{AUTHOR CONTRIBUTIONS}

MM: conceptualization, methodology, formal analysis, investigation, data curation. LV: conceptualization,

\section{REFERENCES}

American Geriatrics Society (2019). AGS Beers Criteria(R) for Potentially Inappropriate Medication Use in Older Adults. (2019). J. Am. Geriatr. Soc., 67(4), 674-694. doi:10.1111/jgs.15767

Arvanitakis, Z., Shah, R. C., and Bennett, D. A. (2019). Diagnosis and Management of Dementia: Review. Jama 322 (16), 1589-1599. doi:10.1001/jama.2019.4782

Byers, A. L., and Yaffe, K. (2011). Depression and Risk of Developing Dementia. Nat. Rev. Neurol. 7 (6), 323-331. doi:10.1038/nrneurol.2011.60

Calsolaro, V., Femminella, G. D., Rogani, S., Esposito, S., Franchi, R., Okoye, C., et al. (2021). Behavioral and Psychological Symptoms in Dementia (BPSD) and the Use of Antipsychotics. Pharmaceuticals 14 (3), 246. doi:10.3390/ ph14030246

Calvo Torres, L. F., Bernal Cobo, R., Trujillo Quintero, P. M., Gaviria Mendoza, A., and Machado Alba, J. E. (2019). Patrones de uso de fármacos antidemencia en un grupo de pacientes de Colombia. Rev. Neurol. 68 (10), 409-416. doi:10. 33588/rn.6810.2018361

Cerejeira, J., Lagarto, L., and Mukaetova-Ladinska, E. B. (2012). Behavioral and Psychological Symptoms of Dementia. Front. Neur. 3, 73. doi:10.3389/fneur. 2012.00073

Collins, J. D., Henley, S. M. D., and Suárez-González, A. (2020). A Systematic Review of the Prevalence of Depression, Anxiety, and Apathy in Frontotemporal Dementia, Atypical and Young-Onset Alzheimer's Disease, and Inherited Dementia. Int. Psychogeriatr. 20, 1-20. [Epub ahead of print]. doi:10.1017/s1041610220001118

Crews, M. P. K., and Howes, O. D. (2012). Is Antipsychotic Treatment Linked to Low Bone mineral Density and Osteoporosis? A Review of the Evidence and the Clinical Implications. Hum. Psychopharmacol. Clin. Exp. 27 (1), 15-23. doi:10. 1002/hup. 1265

Cruz Jentoft, A. J., and Hernández, B. (2014). Rivastigmine as Treatment for Patients with Mild to Moderately Severe Alzheimer Disease under normal Clinical Practice Conditions. The ENTERPRISE Study. Neurología (English Edition), 29(1), 1-10. doi:10.1016/j.nrleng.2013.01.002

Cunningham, E. L., McGuinness, B., Herron, B., and Passmore, A. P. (2015). Dementia. Ulster Med. J. 84 (2), 79-87.

Dell'osso, B., and Lader, M. (2013). Do benzodiazepines Still Deserve a Major Role in the Treatment of Psychiatric Disorders? A Critical Reappraisal. Eur. Psychiatry 28 (1), 7-20. doi:10.1016/j.eurpsy.2011.11.003

Dolder, C. R., and McKinsey, J. (2010). Quetiapine for Sleep in Patients with Dementia. The Consultant Pharmacist 25 (10), 676-679. doi:10.4140/TCP.n. 2010.676

Donoghue, J., and Lader, M. (2010). Usage of Benzodiazepines: A Review. Int. J. Psychiatry Clin. Pract. 14 (2), 78-87. doi:10.3109/13651500903447810

Ferretti, L., McCurry, S. M., Logsdon, R., Gibbons, L., and Teri, L. (2001). Anxiety and Alzheimer's Disease. J. Geriatr. Psychiatry Neurol. 14 (1), 52-58. doi:10. $1177 / 089198870101400111$

Fink, H. A., Linskens, E. J., MacDonald, R., Silverman, P. C., McCarten, J. R., Talley, K. M. C., et al. (2020). Benefits and Harms of Prescription Drugs and Supplements for Treatment of Clinical Alzheimer-type Dementia. Ann. Intern. Med. 172 (10), 656-668. doi:10.7326/m19-3887

Frisoni, G. B., Jack, C. R., Jr., Bocchetta, M., Bauer, C., Frederiksen, K. S., Liu, Y., et al. (2015). The EADC-ADNI Harmonized Protocol for Manual Hippocampal Segmentation on Magnetic Resonance: Evidence of Validity. Alzheimers methodology, data curation, writing original draft. JO: investigation, data curation. ML: investigation, data curation. JM: methodology, validation, formal analysis, resources, writing, review and editing, supervision.

\section{ACKNOWLEDGMENTS}

We thank Soffy Claritza López for her work in obtaining the database.

Dement 11 (2), 111-125. doi:10.1016/j.jalz.2014.05.175610.1016/j.jalz.2014. 05.1761

Goodman, R. A., Lochner, K. A., Thambisetty, M., Wingo, T. S., Posner, S. F., and Ling, S. M. (2017). Prevalence of Dementia Subtypes in United States Medicare Fee-For-Service Beneficiaries, 2011-2013. Alzheimer's Demen. 13 (1), 28-37. doi:10.1016/j.jalz.2016.04.002

Gorelick, P. B., Scuteri, A., Black, S. E., DeCarli, C., Greenberg, S. M., Iadecola, C., et al.(2011). Vascular Contributions to Cognitive Impairment and Dementia. Stroke, 42(9), 2672-2713. doi:10.1161/STR.0b013e3182299496

Hereu, P., and Vallano, A. (2011). Uso de antipsicóticos en pacientes con demencia. Revista Española de Geriatría y Gerontología 46 (1), 50-53. doi:10.1016/j.regg. 2010.11.003

Jalbert, J. J., Eaton, C. B., Miller, S. C., and Lapane, K. L. (2010). Antipsychotic Use and the Risk of Hip Fracture Among Older Adults Afflicted with Dementia. J. Am. Med. Directors Assoc., 11(2), 120-127. doi:10.1016/j.jamda.2009.10.001

Jia, J., Zuo, X., Jia, X.-F., Chu, C., Wu, L., Zhou, A., et al. (2016). Diagnosis and Treatment of Dementia in Neurology Outpatient Departments of General Hospitals in China. Alzheimer's Demen. 12 (4), 446-453. doi:10.1016/j.jalz. 2015.06.1892

Kamble, P., Chen, H., Sherer, J. T., and Aparasu, R. R. (2009). Use of Antipsychotics Among Elderly Nursing Home Residents with Dementia in the US. Drugs \& Aging 26 (6), 483-492. doi:10.2165/00002512-200926060-00005

Kocahan, S., and Doğan, Z. (2017). Mechanisms of Alzheimer's Disease Pathogenesis and Prevention: The Brain, Neural Pathology, N-Methyl-DAspartate Receptors, Tau Protein and Other Risk Factors. Clin. Psychopharmacol. Neurosci. 15 (1), 1-8. doi:10.9758/cpn.2017.15.1.1

Langballe, E. M., Engdahl, B., Nordeng, H., Ballard, C., Aarsland, D., and Selbæk, G. (2014). Short- and Long-Term Mortality Risk Associated with the Use of Antipsychotics Among 26,940 Dementia Outpatients: a Population-Based Study. Am. J. Geriatr. Psychiatry 22 (4), 321-331. doi:10.1016/j.jagp.2013. 06.007

Liao, K.-M., Ho, C.-H., Ko, S.-C., and Li, C.-Y. (2015). Increased Risk of Dementia in Patients with Chronic Obstructive Pulmonary Disease. Medicine (Baltimore) 94 (23), e930. doi:10.1097/md.0000000000000930

Magierski, R., Sobow, T., Schwertner, E., and Religa, D. (2020). Pharmacotherapy of Behavioral and Psychological Symptoms of Dementia: State of the Art and Future Progress. Front. Pharmacol. 11, 1168. doi:10.3389/fphar.2020.01168

Maust, D. T., Kim, H. M., Seyfried, L. S., Chiang, C., Kavanagh, J., Schneider, L. S., et al. (2015). Antipsychotics, Other Psychotropics, and the Risk of Death in Patients with Dementia. JAMA Psychiatry 72 (5), 438-445. doi:10.1001/ jamapsychiatry.2014.3018

Neil, W., Curran, S., and Wattis, J. (2003). Antipsychotic Prescribing in Older People. Age and ageing 32 (5), 475-483. doi:10.1093/ageing/afg061

Nemeroff, C. B. (2005). Use of Atypical Antipsychotics in Refractory Depression and Anxiety. J. Clin. Psychiatry, 66 (Suppl 8), 13-21. Retrieved from http:// europepmc.org/abstract/MED/16336032. (Accessed January 7, 2021).

Neville, C., and Teri, L. (2011). Anxiety, Anxiety Symptoms, and Associations Among Older People with Dementia in Assisted-Living Facilities. Int. J. Ment. Health Nurs. 20 (3), 195-201. doi:10.1111/j.1447-0349.2010.00724.x

O'Mahony, D., O’Sullivan, D., Byrne, S., O’Connor, M. N., Ryan, C., and Gallagher, P. (2014). STOPP/START Criteria for Potentially Inappropriate Prescribing in Older People: Version 2. Age and ageing 44 (2), 213-218. doi:10.1093/ageing/ afu 145 
Peng, Y.-H., Wu, B.-R., Su, C.-H., Liao, W.-C., Muo, C.-H., Hsia, T.-C., et al. (2015). Adult Asthma Increases Dementia Risk: a Nationwide Cohort Study. J. Epidemiol. Community Health 69 (2), 123-128. doi:10.1136/jech-2014204445

Prince, M., Bryce, R., Albanese, E., Wimo, A., Ribeiro, W., and Ferri, C. P. (2013). The Global Prevalence of Dementia: a Systematic Review and Metaanalysis. Alzheimer's Demen. 9 (1), 63-75. doi:10.1016/j.jalz.2012.11.007

Rayner, A. V., O’Brien, J. G., Schoenbachler, B., and Shoenbachler, B. (2006). Behavior Disorders of Dementia: Recognition and Treatment. Am. Fam. Physician 73 (4), 647-652.

Rizzi, L., Rosset, I., and Roriz-Cruz, M. (2014). Global Epidemiology of Dementia: Alzheimer's and Vascular Types. Biomed. Res. Int. 2014, 1-8. doi:10.1155/2014/ 908915

Román, D. M. J. (2011). Psychiatric Disorder Induced by Medications. Rev. Cient Cienc Méd 14, 21-24.

Rosano, C., Naydeck, B., Kuller, L. H., Longstreth, W. T., Jr., and Newman, A. B. (2005). Coronary Artery Calcium: Associations with Brain Magnetic Resonance Imaging Abnormalities and Cognitive Status. J. Am. Geriatr. Soc. 53 (4), 609-615. doi:10.1111/j.1532-5415.2005.53208.x

Schneider, L. S., Dagerman, K., and Insel, P. S. (2006a). Efficacy and Adverse Effects of Atypical Antipsychotics for Dementia: Meta-Analysis of Randomized, Placebo-Controlled Trials. Am. J. Geriatr. Psychiatry 14 (3), 191-210. doi:10. 1097/01.JGP.0000200589.01396.6d

Schneider, L. S., Tariot, P. N., Dagerman, K. S., Davis, S. M., Hsiao, J. K., Ismail, M. S., et al. (2006b). Effectiveness of Atypical Antipsychotic Drugs in Patients with Alzheimer's Disease. N. Engl. J. Med. 355 (15), 1525-1538. doi:10.1056/ NEJMoa061240

Schulze, J., van den Bussche, H., Glaeske, G., Kaduszkiewicz, H., Wiese, B., and Hoffmann, F. (2013). Impact of Safety Warnings on Antipsychotic Prescriptions in Dementia: Nothing Has Changed but the Years and the
Substances. Eur. Neuropsychopharmacol., 23(9), 1034-1042. doi:10.1016/j. euroneuro.2013.02.001

Shen, H., Li, R., Yan, R., Zhou, X., Feng, X., Zhao, M., et al. (2018). Adjunctive Therapy with Statins in Schizophrenia Patients: A Meta-Analysis and Implications. Psychiatry Res., 262, 84-93. doi:10.1016/j.psychres.2018. 02.018

Valladales-Restrepo, L. F., Duran-Lengua, M., and Machado-Alba, J. E. (2019). Potentially Inappropriate Prescriptions of Anticholinergics Drugs in Alzheimer's Disease Patients. Geriatr. Gerontol. Int. 19 (9), 913-917. doi:10. 1111/ggi.13748

Vina Latin Small Letter, S. W. C. B. I. R., Buciuta, A., and Coman, H. G. (2021). Atypical Antipsychotics in the Treatment of Psychotic Symptoms in Alzheimer's Disease: a Systematic Review. Int. Clin. Psychopharmacol. [Epub ahead of print]. doi:10.1097/yic.0000000000000358

Zhong, W., Cruickshanks, K. J., Schubert, C. R., Acher, C. W., Carlsson, C. M., Klein, B. E. K., et al. (2012). Carotid Atherosclerosis and 10-year Changes in Cognitive Function. Atherosclerosis 224 (2), 506-510. doi:10.1016/j. atherosclerosis.2012.07.024

Conflict of Interest: The authors declare that the research was conducted in the absence of any commercial or financial relationships that could be construed as a potential conflict of interest.

Copyright (c) 2021 Machado-Duque, Valladales-Restrepo, Ospina-Cano, LondoñoSerna and Machado-Alba. This is an open-access article distributed under the terms of the Creative Commons Attribution License (CC BY). The use, distribution or reproduction in other forums is permitted, provided the original author(s) and the copyright owner(s) are credited and that the original publication in this journal is cited, in accordance with accepted academic practice. No use, distribution or reproduction is permitted which does not comply with these terms. 